\title{
The European and Japanese outbreaks of H5N8 derive from a single source population providing evidence for the dispersal along the long distance bird migratory flyways
}

Andrew Dalby, Munir Iqbal

The origin of recent parallel outbreaks of the high pathogenicity H5N8 avian flu virus in Europe and in Japan can be traced to a single source population, which has most likely been spread by migratory birds. By using Bayesian coalescent methods to analyze the DNA sequences of the virus to find the times for divergence and combining this sequence data with bird migration data we can show the most likely locations and migratory pathways involved in the origin of the current outbreak. This population was most likely located in the Siberian summer breeding grounds of long-range migratory birds. These breeding grounds provide a connection between different migratory flyways and explain the current outbreaks in remote locations. By combining genetic methods and epidemiological data we can rapidly identify the sources and the dispersion pathways for novel avian influenza outbreaks. 
2 Andrew R. Dalby

3 Faculty of Science and Technology, University of Westminster, 115 New Cavendish Street,

4 Westminster, W1W 6UW, UK.

5 Munir Iqbal

6 Avian Viral Diseases Programme, The Pirbright Institute, Compton Laboratory, Newbury, Berkshire.

7 RG20 7NN, UK

8

9 A.Dalby@westminster.ac.uk 
11 Introduction

12 The H5N8 subtype of influenza A is a comparatively rare influenza A subtype that was first isolated

13 from a turkey in Ireland in 1983 (Murphy 1986). After that initial outbreak there were no more cases of

14 H5N8 until 2001 when a case was identified during environmental monitoring in a wild bird in New

15 Jersey. Since then there have been a few sporadic detections in the United States but the biggest single

16 outbreak to date has been in Korea in January 2014 (Lee et al. 2014).

18 This outbreak was preceded by cases in Eastern China in 2010 that are distinct from the American

19 virus. Although the Korean outbreak strains had the same subtype the Asian outbreak seems to have

20 been the product of a re-assortment of viral segments from other $\mathrm{H} 5$ containing subtypes such as $\mathrm{H} 5 \mathrm{~N} 1$

21 or $\mathrm{H} 5 \mathrm{~N} 5$ and an $\mathrm{N} 8$ containing subtype, rather than from the evolution of the previous H5N8 lineages

22 (Lee et al. 2014; Zhao et al. 2013). The Korean outbreak has been subdivided into two lineages one of

23 which is closely related to the Chinese sequences and that has only been identified in two samples in

24 Gochang and a second that contains all the other cases and that was originally identified in Buan (Fan

25 et al. 2014; Jeong et al. 2014).

27 The H5N8 virus is an example of a highly pathogenic avian influenza A (HPAI). These HPAI viruses

28 pose a significant threat to domestic poultry as mortality rates amongst chickens are particularly high

29 and can reach $100 \%$. Recent studies of the virus have shown that it has a pathogenicity index of 3 in

30 chickens (Kim et al. 2014). This is significantly higher than that of the original H5N8 from Ireland

31 although that is a distinct lineage (Alexander et al. 1986). The management of the outbreak in Korea in

32 early 2014 resulted in the culling of over 10 million birds or $6 \%$ of the total Korean poultry flock 
33 (Kang). Ducks and particularly wild ducks such as mallards are often asymptomatic but can still be

34 carriers of the H5N8 virus (Bae et al. 2014; Kang; Kim et al. 2014).

36 Currently unlike H5N1, H5N8 is not considered a threat to human health as there has not been a case

37 of transmission to humans. However this might be a result of the low incidence of the subtype as

38 studies have shown that it can be transmitted to ferrets and mice, and antibodies have been detected in

39 domestic dogs (Kim et al. 2014). The results of genetic analysis of the H5N8 virus in infected ferrets

40 have also shown that mutations to a mammalian transmissible form occur rapidly.

42 In November 2014 H5N8 was detected in Europe with outbreaks in poultry farms in the Netherlands,

43 Germany and the United Kingdom. At the same time the virus was also detected in farmed birds and

44 wild birds in Japan. This study identifies the probable geographical source and pathway for dispersal of 45 the November/December outbreaks of H5N8. 
47 Materials and Methods

48 The complete set of available H5N8 nucleotide sequences were downloaded from the NCBI Influenza

49 Virus Resource and GISAID (Bao et al. 2008; Bogner et al. 2006). The search was restricted to

50 complete sequences of $\mathrm{H} 5 \mathrm{~N} 8$ within the NCBI influenza virus resource.

52 All of the sequences were aligned with Muscle v3.8.31 (Edgar 2004). Manual inspection and editing of

53 the sequences was carried out using Mega6.06 (Tamura et al. 2013). During manual editing the 5' end

54 of the sequence was edited to remove the un-translated region. All sequences begin at the start codon.

55 Sequences with missing nucleotides were removed. This included 3 partial Chinese duck sequences.

56 There was no editing at the 3 ' end of the nucleotide sequences as influenza uses a variety of stop

57 codons that are sometimes repeated. Tip dates were assigned according to the year of collection.

59 A subset of the sequences was created for the detailed analysis of the hemagglutinin and neuraminidase

60 sequences containing only the sequences from 2014. This provides a more detailed analysis for

61 calculating the divergence dates from the Korean outbreak. For these calculations tip dates were given

62 in months before December 2014. This solves the problem of missing data from earlier sequences

63 where months might not be available.

65 Bayesian Coalescent trees were calculated for all the different segments and the subsets using

66 Beast2.1.3 (Bouckaert et al. 2014). The model used assumed an exponential population growth and tip

67 dates were set from the sequence collection dates. The Hasegawa-Kishino-Yano (HKY) nucleotide 
68 substitution model was used with an assumed strict molecular clock (Hasegawa et al. 1985) as using a

69 rigid clock was shown to give the best effective sample size when compared to other clock models,

70 which gave similar parameter values but also showed significant auto-correlation. The use of the HKY

71 model in preference to Tamura-Nei in preference to Tamura-Nei was also supported by analysis using

72 Model-Test (Posada \& Crandall 1998). All the final simulations were performed as a single run with a

73 minimum of 10 million iterations except for the PA segment that needed 20 million, PB2 segment that

74 needed 30 million and NS segment that needed 40 million iterations to achieve a suitable level of chain

75 sampling. A burn in period of $10 \%$ was used for all samples.

77 Analysis of the Bayesian coalescent output was carried out using Tracer1.6.0 (Rambaut \& Drummond

78 2013a). All simulations were run until the effective sample sizes for all of the parameters in the model

79 were over 200. The maximum clade credibility trees were calculated using Treeannotater 2.1.2 along

80 with the median node heights and the final tree diagrams were generated using FigTree1.4.2 (Rambaut

81 2007; Rambaut \& Drummond 2013b).

83 All of the available H5 hemagglutinin subunit and N8 neuraminidase subunits were downloaded from

84 the NCBI Influenza Virus Resource and GISAID (Bao et al. 2008; Bogner et al. 2006). This data was

85 used to carry out a complete phylogenetic analysis for each of the segments. The sequences were

86 initially aligned using MAFFT (Katoh \& Standley 2013). FastTree2.1 was used to create an

87 approximate maximum likelihood tree for all of the sequences. The resulting trees were visualised and

88 annotated with FigTree 1.4.2 (Rambaut \& Drummond 2013a) 
89 All of the XML files used to calculate the trees for each of the segments and for the 2014

90 hemagglutinin and neuraminidase trees are available as supplementary materials as are the tree files

91 they produce. The tree files for the complete $\mathrm{H} 5$ and $\mathrm{N} 8$ segment analysis is also available in the

92 supplementary materials.

93

94 The locations of the H5N8 cases were taken from the EMPRES Global Animal Disease Information

95 System (EMPRES-i) and information about the original reports were sourced from the Avian Flu Diary

96 Blog (http://afludiary.blogspot.co.uk/). The map was created using Google maps and is available from:

97 https://www.google.com/maps/d/edit?mid=zcvUWKLLjKsE.kvYJ1NxAer8k 
99 Results and Discussion

100 The Bayesian coalescent analysis of the complete set of $\mathrm{H} 5 \mathrm{~N} 8$ sequences produces a consistent gene

101 tree, where the same clade structure is produced for all eight segments (supplementary figures 1 to 8 ).

102 The current Korean outbreak is the product of a recent viral re-assortment and so complete trees of the

103 H5 subunits from all of the viral subtypes and of the N8 subunits from all of the viral subtypes were

104 created to confirm that there had not been another re-assortment between the European and Japanese

105 sequences. These results show that the European and Japanese sequences all form a single cluster

106 closely related to, but distinct from those found in the Korean outbreak. This clustering suggests that

107 the viruses are likely to come from a single source population.

109 Bayesian coalescent analysis of the 2014 sequences also permits the sequence divergence time to be

110 calculated with greater accuracy (Lemey et al. 2009). The Bayesian coalescent trees for the 2014

111 hemaggluinin gene segments and the neuraminidase gene segments are shown in figures 1 and 2 . The

112 bars above the branch points represent the $95 \%$ highest posterior density for the age of that node. The

$113 \mathrm{x}$-axis represents the date in months before November 2014. For the viral hemagglutinin gene segment

114 the cluster of sequences responsible for the current European and Japanese outbreaks diverged between

115 a median value of 2.65 and 6.57 months before November 2014 (95\% highest posterior density

116 interval). Only the hemagglutinin and neuraminidase segments are available from the infected German

117 turkey and the median divergence time calculated from the neuraminidase tree is from 1.39 to 6.21

118 months before November 2014 (95\% highest posterior density interval). This is in good agreement

119 with the results from the hemagglutinin tree and shows that the Japanese and European sequences

120 diverged after the last reported cases of the Korean outbreak. 
122 During the Korean outbreak a large number of wild birds were also affected, particularly in the region

123 around the Dong-Lim reservoir (Jeong et al. 2014). One of the bird species that was found to be

124 infected was the Baikal Teal (Anas formosa) which is a migratory species that over- winters in Korea

125 before returning to North Eastern Siberia to breed during the summer months (Allport et al. 1991). This

126 migration coincided with the last Korean $\mathrm{H} 5 \mathrm{~N} 8$ sequences identified in wild birds during the initial

127 outbreak. This migration also falls within the range of divergence dates from the Bayesian coalescent

128 analysis for the current cluster of H5N8 cases in Europe. This result strongly suggests that the virus

129 was carried to the Siberian breeding grounds as the Baikal teal migrated north and that the European

130 and Japanese sequences evolved there.

132 The wide geographic dispersal of the current outbreaks gives further support to the contention that

133 migratory birds are the source of the virus. Most of the recent cases occur close to the coastline and in

134 areas where there are lakes and known sites for waterfowl and migratory birds. In Holland the virus has

135 been identified in widgeon and in Germany it was found in a common teal (Anas crecca) that had no

136 apparent clinical symptoms. The Japanese have recently identified the wild bird species infected with

137 the virus as tundra swans (Cygnus columbianus), white naped cranes (Grus vipio), pochards (Aythya

138 ferina) and wild ducks (Anas platyrhyncus).

140 The breeding grounds and migratory staging grounds for Baikal teal overlap with those for many other

141 migratory species including common species such as mallards, pochards, widgeon (Anas penelope),

142 common teal, whooper swans (Cygnus cygnus) and tundra swans, as well as endangered species such 
143 as white-naped cranes (Miyabayashi \& Mundkur 1999). Mallard and teal have previously been

144 identified as having a high prevalence (between 6 and 7\%) for influenza A virus (Munster et al. 2007).

145 The bird migrations flow from Siberia along the five different flyways that overlap in Central Siberia.

146 They are the East Atlantic, East Asia Australian, East Africa West Asia, Central Asia and Black Sea

147 Mediterranean flyways. So far H5N8 infections in birds have only been detected in the East Atlantic

148 and East Asian Australian flyways (figure 9). The absence from other flyways can be explained either

149 through transmission by a limited number of bird migratory species, or because or the lack of

150 surveillance in these geographical regions. Although there have been a few cases recently reported in

151 North America these are from the different lineage not related to the Korean outbreak.

152

153 This year the winter migration has been later than usual because of the warmer autumn weather. Ideally

154 satellite-tracking data would be available for all of the migrating species from their summer breeding

155 grounds. However tracking data is only available for species of interest that include Bewick swans

156 (Cygnus bewickii), a sub-species of Tundra swans. These tracking data show that their migration was

157 delayed until late October and early November, which coincided with the European outbreaks of the

158 H5N8 virus (Slimbridge Wildlife Trust).

160 Gaidet and co-workers considering the spread of another HPAI, H5N1 suggested that the risk of

161 transmission by migratory birds was only a low risk because of the need for asymptomatic infections

162 and also taking into account the distances travelled, the time taken and the number of staging points

163 along the journey (Gaidet et al. 2010). However low pathogenicity avian influenza have been shown to

164 spread via migrating birds because the large majority of cases remain asymptomatic (Dusek et al. 2014; 
165 Lam et al. 2012). In the case of high pathogenicity H5N8, the virus has been shown to be

166 asymptomatic in mallards, and there would have been selection of virus variants that are asymptomatic

167 amongst the Baikal teal if the disease has been carried by a migrant bird (Bae et al. 2014; Kang ; Kim

168 et al. 2014). Dispersion of the virus through migratory flyways still requires that there is relay infection

169 for the virus to spread over very long migratory distances.

171 Previous studies had shown that there was a spatio-temporal relationship between bird migration and

172 the spread of the HPAI H5N1 subtype (Takekawa et al. 2010). However it was not possible to show

173 that transmission by the migratory birds was the cause of this correlation. In this case the genetic data

174 and the calculated divergence times show that the evolutionary events responsible for generating the

175 European and Japanese cases occurred in the summer months in a single location.

176

177 The initial outbreak affected a large number of birds during the period close to the main spring

178 migration this increased the likelihood of long-range transmission. The spread of the virus requires that

179 there is relay infection so that it spreads amongst susceptible birds at the migratory staging points, in

180 order to provide the next step in transmission. This is seen with the presence of an increased number of

181 cases at staging points such as the Netherlands. This is supported by the current limited amount of data,

182 although there are other staging points in Estonia and in Denmark where there have not been any

183 reported cases (Beekman et al. 2002; Green et al. 2002).

\section{Conclusions}


186 The results presented here give strong support to the view that the H5N8 outbreaks that occurred in

187 Europe and Japan in December 2014 originated from a single source population. Although there is no

188 direct evidence of what this source population was it is likely that the virus was spread along long-

189 range migratory routes as trade is a less likely source given the absence in disease infections during the

190 summer months. This suggests that the summer breeding grounds for migratory species such as Baikal

191 teal are the most likely geographical location for the source of the outbreaks.

192 Increased monitoring for HPAI is needed in areas where there is overlap between migrating species,

193 especially if this zone links very disparate geographical regions. This could be achieved through

194 environmental monitoring of faecal samples in areas where migratory birds congregate. In the case of

195 H5N8 the main costs are economic as it is not currently a human pathogenic subtype, but it has had

196 devastating consequences for the Korean poultry industry. However the longer the virus is in

197 circulation in wild birds and poultry the more likely it is that a human case will occur, especially

198 considering the close relationship to the H5N1 strains and existing evidence that shows the virus can

199 reproduce in mammalian hosts.

201 It is important to involve local communities and experts as well as farmers so that we can significantly

202 improve the monitoring network giving earlier warning of potential epidemics. This also means

203 improved communication between international organizations and making the biological sequence data 204 available in a timely manner.

\section{Acknowledgments}


208 Special thanks must go to Michael Coston whose Avian Flu Diary Blog has been an important source

209 of information for the current outbreak. Also thanks to David Welch for his comments on the Bayesian

210 analysis. Finally thanks to the editor Prof. Claus Wilke and the anonymous referee who have pointed

211 us towards a previously unexplored area for our research.

212 


\section{Figure Legends}

215 Figure 1: Bayesian coalescent gene tree for hemagglutinin

217 Bayesian coalescent gene tree for the 2014 H5N8 hemagglutinin sequences. The blue bars on the nodes 218 represent the $95 \%$ highest posterior density intervals of the branch ages. The European and Japanese 219 clade is highlighted in red. 0 on the x-axis represents November 2014.

Figure 2: Bayesian coalescent tree for neuraminidase

Bayesian coalescent gene tree for the 2014 H5N8 neuraminidase sequences. The blue bars on the nodes represent the $95 \%$ highest posterior density intervals of the branch ages. The European and Japanese clade is highlighted in red. 0 on the $\mathrm{x}$-axis represents November 2014.

Figure 3: Migratory flyways and cases of H5N8.

Bird migratory flyways and the December 2014 cases of H5N8 (Boere \& Stroud 2006). The Eastern Asian Australian flyway is in red. The East Atlantic flyway is in dark blue. An expandable version of this map is available from:

https://www.google.com/maps/d/viewer?mid=zcvUWKLLjKsE.kvYJ1NxAer8k - Map Data (C) 2015

Google, INEGI 


\section{References}

237

238

239

240

241

242

243

244

245

246

247

248

249

250

251

252

253

254

255

256

257

258

259

260

261

262

263

264

265

266

267

268

269

270

271

272

273

274

275

276

277

278

279

280

281

282

Alexander D, Parsons G, and Manvell R. 1986. Experimental assessment of the pathogenicity of eight avian influenza A viruses of $\mathrm{H} 5$ subtype for chickens, turkeys, ducks and quail. Avian pathology 15:647-662.

Allport G, Poole C, Park E, Jo S, and Eldridge M. 1991. The feeding ecology, requirements and distribution of Baikal Teal Anas formosa in the Republic of Korea. Wildfowl 42:98-107.

Bae Y, Lee S-B, Min KC, Mo J-S, Jeon EO, Koo B-S, Kwon H-I, Choi YK, Kim JJ, and Kim J-N. 2014. Pathological evaluation of natural cases of a highly pathogenic avian influenza virus, subtype H5N8, in broiler breeders and commercial layers in South Korea. Avian Diseases.

Bao Y, Bolotov P, Dernovoy D, Kiryutin B, Zaslavsky L, Tatusova T, Ostell J, and Lipman D. 2008. The influenza virus resource at the National Center for Biotechnology Information. Journal of virology 82:596-601.

Beekman JH, Nolet BA, and Klaassen M. 2002. Skipping swans: fuelling rates and wind conditions determine differential use of migratory stopover sites of Bewick's Swans Cygnus bewickii. Ardea 90:437-460.

Birdlife International. Baikal Teal Sibirionetta formosa. Available at http://www.birdlife.org/datazone/species/factsheet/22680317 (accessed 4th December 2014).

Bogner P, Capua I, Lipman DJ, and Cox NJ. 2006. A global initiative on sharing avian flu data. Nature 442:981981.

Bouckaert R, Heled J, Kühnert D, Vaughan T, Wu C-H, Xie D, Suchard MA, Rambaut A, and Drummond AJ. 2014. BEAST 2: a software platform for Bayesian evolutionary analysis. PLoS computational biology 10:e1003537.

Dusek RJ, Hallgrimsson GT, Ip HS, Jónsson JE, Sreevatsan S, Nashold SW, TeSlaa JL, Enomoto S, Halpin RA, and Lin X. 2014. North Atlantic Migratory Bird Flyways Provide Routes for Intercontinental Movement of Avian Influenza Viruses. PloS one 9:e92075.

Edgar RC. 2004. MUSCLE: multiple sequence alignment with high accuracy and high throughput. Nucleic acids research 32:1792-1797.

Fan S, Zhou L, Wu D, Gao X, Pei E, Wang T, Gao Y, and Xia X. 2014. A novel highly pathogenic H5N8 avian influenza virus isolated from a wild duck in China. Influenza Other Respir Viruses 8:646-653.

Gaidet N, Cappelle J, Takekawa JY, Prosser DJ, Iverson SA, Douglas DC, Perry WM, Mundkur T, and Newman SH. 2010. Potential spread of highly pathogenic avian influenza H5N1 by wildfowl: dispersal ranges and rates determined from large-scale satellite telemetry. Journal of Applied Ecology 47:1147-1157.

Green M, Alerstam T, Clausen P, Drent R, and Ebbinge BS. 2002. Dark-bellied Brent Geese Branta bernicla bernicla, as recorded by satellite telemetry, do not minimize flight distance during spring migration. Ibis 144:106-121.

Hasegawa M, Kishino H, and Yano T-a. 1985. Dating of the human-ape splitting by a molecular clock of mitochondrial DNA. Journal of molecular evolution 22:160-174.

Jeong J, Kang H-M, Lee E-K, Song B-M, Kwon Y-K, Kim H-R, Choi K-S, Kim J-Y, Lee H-J, and Moon O-K. 2014. Highly pathogenic avian influenza virus (H5N8) in domestic poultry and its relationship with migratory birds in South Korea during 2014. Veterinary microbiology 173:249-257.

Kang H-M. Novel Reassortant Influenza A (H5N8) Viruses among Inoculated Domestic and Wild Ducks, South Korea, 2014.

Katoh K, and Standley DM. 2013. MAFFT multiple sequence alignment software version 7: improvements in performance and usability. Molecular biology and evolution 30:772-780.

Kim Y-I, Pascua PNQ, Kwon H-I, Lim G-J, Kim E-H, Yoon S-W, Park S-J, Kim SM, Choi E-J, and Si Y-J. 2014. Pathobiological features of a novel, highly pathogenic avian influenza A (H5N8) virus. Emerging Microbes \& Infections 3:e75.

Lam TTY, Ip HS, Ghedin E, Wentworth DE, Halpin RA, Stockwell TB, Spiro DJ, Dusek RJ, Bortner JB, and Hoskins J. 2012. Migratory flyway and geographical distance are barriers to the gene flow of influenza virus among North American birds. Ecology letters 15:24-33. 
Lee Y-J, Kang H-M, Lee E-K, Song B-M, Jeong J, Kwon Y-K, Kim H-R, Lee K-J, Hong M-S, and Jang I. 2014. Novel reassortant influenza A (H5N8) viruses, South Korea, 2014. Emerging infectious diseases 20:1087. Lemey P, Rambaut A, Drummond AJ, and Suchard MA. 2009. Bayesian phylogeography finds its roots. PLoS computational biology 5:e1000520.

Miyabayashi Y, and Mundkur T. 1999. Atlas of key sites for Anatidae in the East Asian Flyway: Wetlands International.

Munster VJ, Baas C, Lexmond P, Waldenström J, Wallensten A, Fransson T, Rimmelzwaan GF, Beyer WE, Schutten M, and Olsen B. 2007. Spatial, temporal, and species variation in prevalence of influenza $A$ viruses in wild migratory birds. PLoS pathogens 3:e61.

Murphy T. 1986. The control and epidemiology of an influenza A outbreak in Ireland. Acute Virus Infections of Poultry: Springer, 23-28.

Posada D, and Crandall KA. 1998. Modeltest: testing the model of DNA substitution. Bioinformatics 14:817-818.

Rambaut A. 2007. FigTree, a graphical viewer of phylogenetic trees. See http://tree bio ed ac uk/software/figtree.

Rambaut A, and Drummond A. 2013a. Tracer v1. 5 Available from http://beast. bio. ed. ac. uk/Tracer. Accessed. Rambaut A, and Drummond A. 2013b. TreeAnnotator v1. 7.0.

Slimbridge Wildlife Trust. Bewick's Swan Diary. Available at http://www.wwt.org.uk/news/allnews/2014/10/wwt-slimbridge-diaries/wwt-slimbridge-diaries-bewicks-swan-diary/bewicks-reachbritain/ (accessed 4th December 2014).

Takekawa JY, Newman SH, Xiao X, Prosser DJ, Spragens KA, Palm EC, Yan B, Li T, Lei F, and Zhao D. 2010. Migration of waterfowl in the East Asian flyway and spatial relationship to HPAI H5N1 outbreaks. Avian diseases 54:466-476.

Tamura K, Stecher G, Peterson D, Filipski A, and Kumar S. 2013. MEGA6: molecular evolutionary genetics analysis version 6.0. Molecular biology and evolution 30:2725-2729.

Zhao K, Gu M, Zhong L, Duan Z, Zhang Y, Zhu Y, Zhao G, Zhao M, Chen Z, and Hu S. 2013. Characterization of three H5N5 and one H5N8 highly pathogenic avian influenza viruses in China. Veterinary microbiology 163:351-357. 
Bayesian coalescent gene tree for hemagglutinin

Bayesian coalescent gene tree for the 2014 H5N8 hemagglutinin sequences. The blue bars on the nodes represent the $95 \%$ highest posterior density intervals of the branch ages. The European and Japanese clade is highlighted in red. 0 on the $\mathrm{x}$-axis represents November 2014.

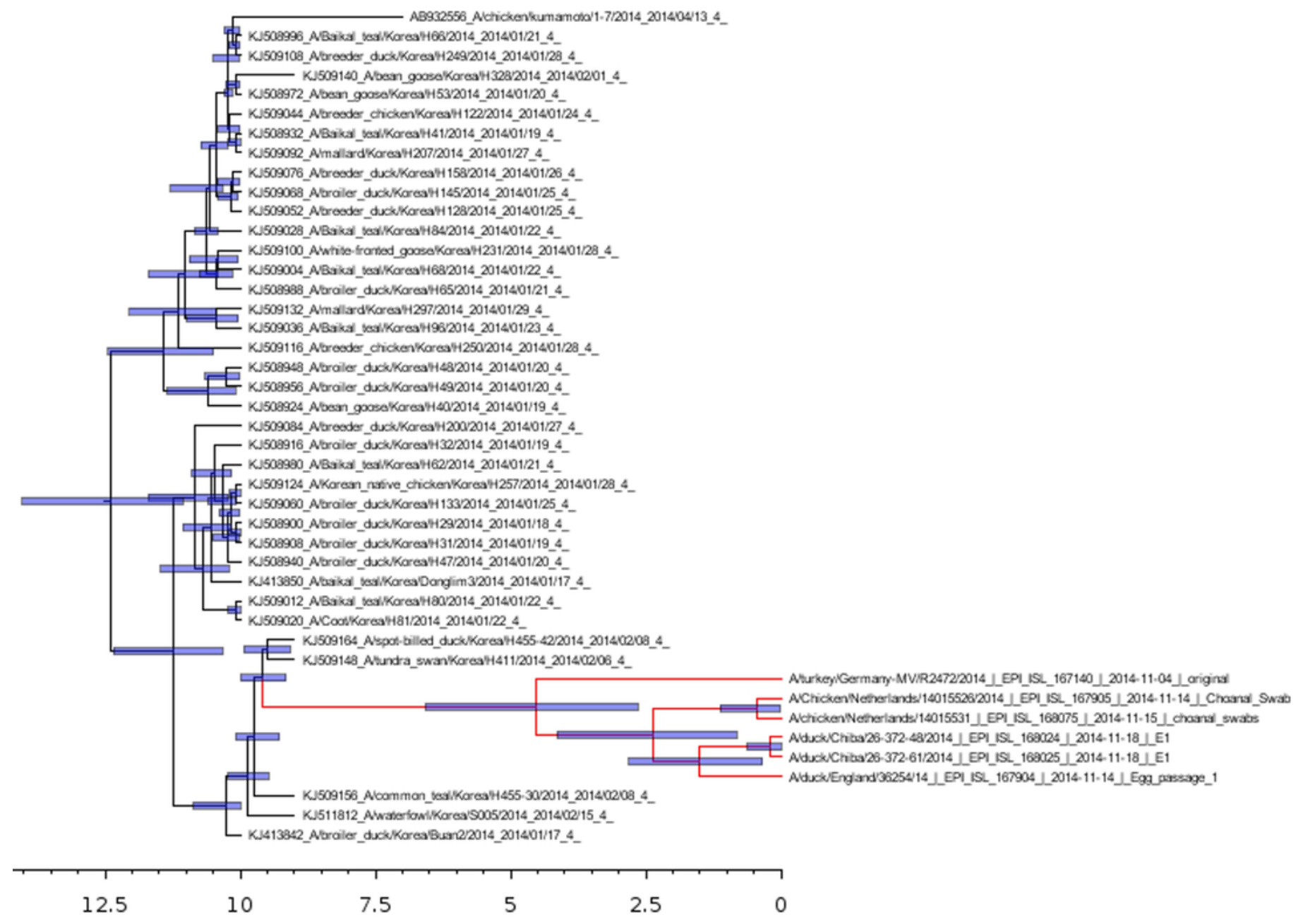


Bayesian coalescent tree for neuraminidase

Bayesian coalescent gene tree for the 2014 H5N8 neuraminidase sequences. The blue bars on the nodes represent the $95 \%$ highest posterior density intervals of the branch ages. The European and Japanese clade is highlighted in red. 0 on the $\mathrm{x}$-axis represents November 2014.

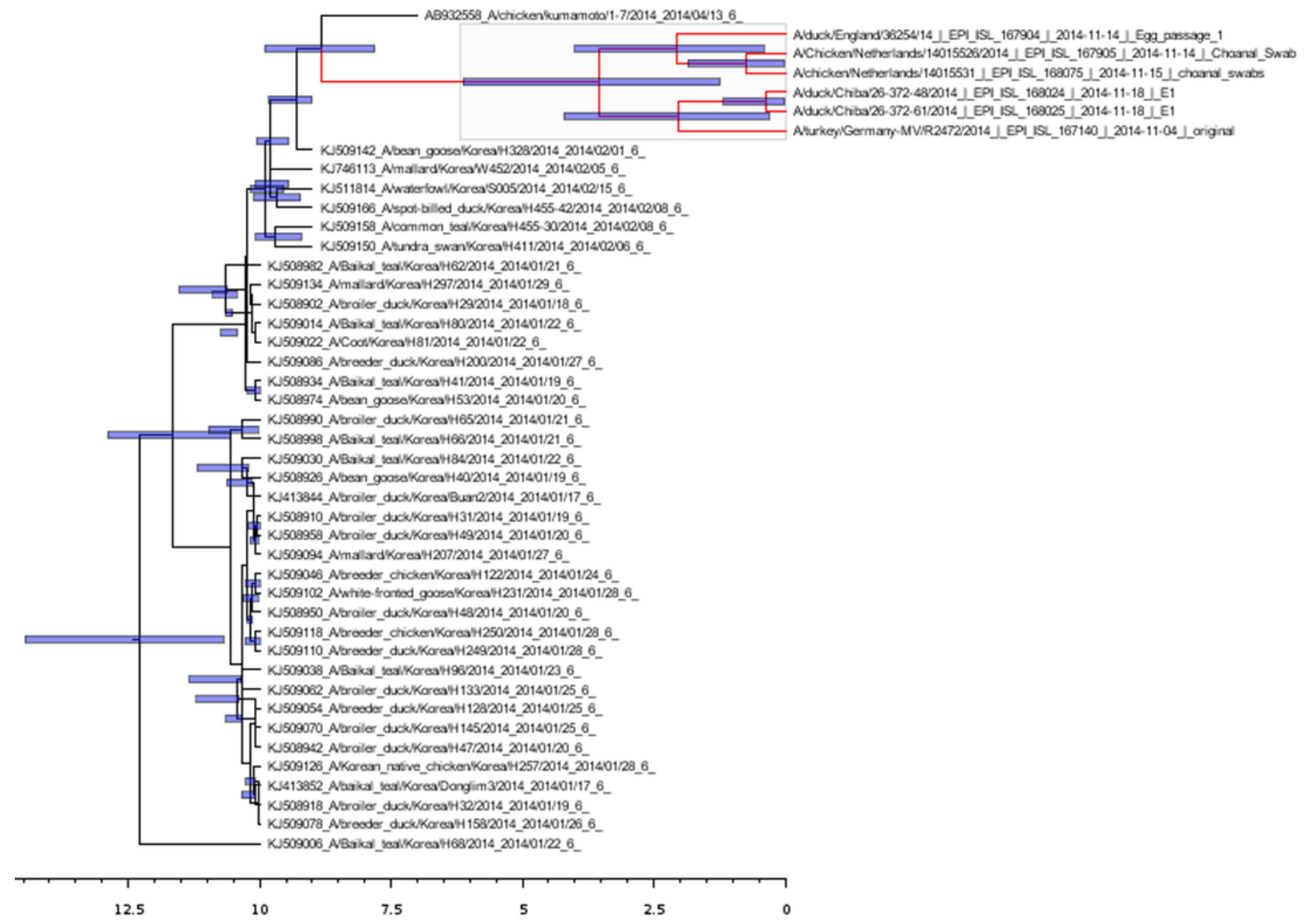


Figure 3 (on next page)

Migratory flyways and cases of H5N8.

Bird migratory flyways and the December 2014 cases of H5N8 (Boere \& Stroud 2006). The Eastern Asian Australian flyway is in red. The East Atlantic flyway is in dark blue. An expandable version of this map is available from:

https://www.google.com/maps/d/viewer?mid=zcvUWKLLjKsE.kvYJ1NxAer8k - Map Data (C) 2015 Google, INEGI 


\section{PeerJ Reviewing Manuscript}

\section{H5N8}

H5N8 cases

All items

Flyways

8 East Atlantic Flyway

$\$$

East Asian Australian Flyway

Map of H5N8 cases December 2014

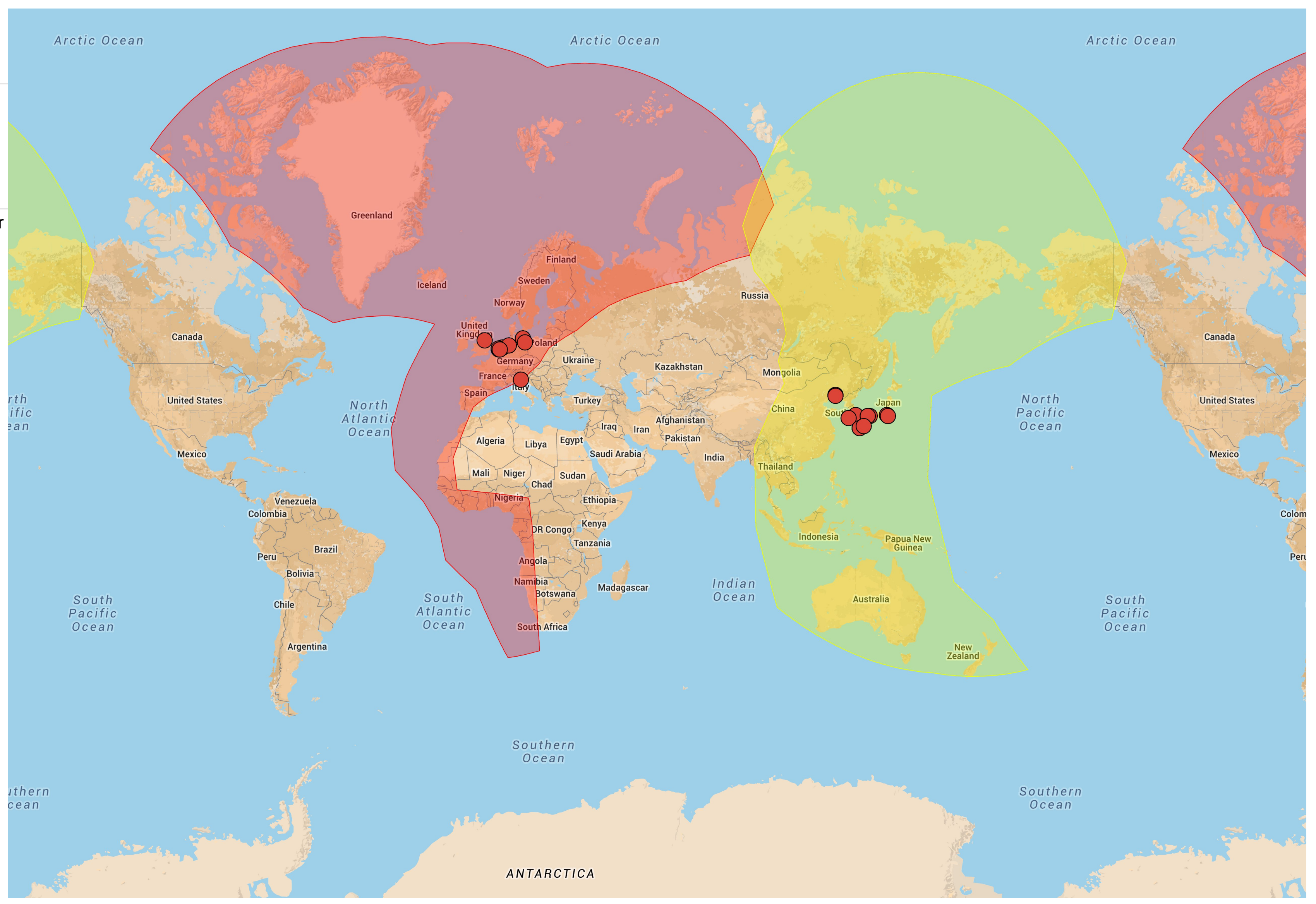

